\title{
Mathematical Modeling of Shock Waves in Inhomogeneous Viscoelastic Two-Component Media
}

\author{
Victor Polenov ${ }^{1}$, Anatoli Chigarev² \\ ${ }^{1}$ Department of Mathematics, Voronezh State University, Voronezh, Russia \\ ${ }^{2}$ Department of Theoretical Mechanics, Belarusian National Technical University, Minsk, Belarus \\ Email: polenov.vrn@mail.ru
}

How to cite this paper: Polenov, V. and Chigarev, A. (2018) Mathematical Modeling of Shock Waves in Inhomogeneous Viscoelastic Two-Component Media. Journal of Applied Mathematics and Physics, 6, 997-1005.

https://doi.org/10.4236/jamp.2018.65086

Received: March 30, 2018

Accepted: May 19, 2018

Published: May 22, 2018

Copyright (c) 2018 by authors and Scientific Research Publishing Inc. This work is licensed under the Creative Commons Attribution International License (CC BY 4.0).

http://creativecommons.org/licenses/by/4.0/

\begin{abstract}
Mathematical modeling of two-component media with a saturated liquid began over 90 years with studies of the consolidation of soils. Two-component must be taken into account when solving a significant number of applied problems arising in various areas of human activity (soils, foams, various cement mortars, sand, porous ceramics, porous sintered composite materials, etc.). Two-component media are widely used in the national economy. For example, in the construction of new airfields and the restoration of destroyed, where the building materials used contain a significant number of voids. The study of wave processes is also very important for the development of new diagnostic methods, new technologies for creating two-component environments that could be applied in the field of engineering, construction, instrumentation, metallurgy, nuclear power and the defense capability of the country. However, the complexity of describing the effects of the interaction of components, heat transfer, and other related processes has led to the fact that until now the generally accepted models (elastic medium-liquid) for a fluid-saturated two-component medium have not been fully developed. Therefore, it is of interest to develop a mathematical two-component model when one of the components represents an inhomogeneous viscoelastic medium and the other is a compressible fluid. The presence and degree of porosity in materials is accounted for by a porosity coefficient equal to the ratio of the pore volume to the total volume occupied by the medium.
\end{abstract}

\section{Keywords}

Wave Propagation, Porous Medium, Dynamic Quantities, Elasticity, Viscoelastic, Fluid 


\section{Introduction}

The propagation of shock waves in an isotropic viscoelastic homogeneous and inhomogeneous medium was considered in [1] [2] [3]. The nature of the damping of waves in such media is determined by the geometry of the discontinuity surface and the rheological model described by the corresponding relaxation nuclei.

It is of interest to consider shock waves in a fluid-saturated inhomogeneous two-component medium when one of the components is viscoelastic. Porosity [4] [5] [6] is understood to be effective porosity, taking into account only interconnected pores. Isolated pores are considered as elements of the viscoelastic part of the porous skeleton. It is assumed that the pore sizes are small in comparison with the distance at which the kinematic and geometric characteristics of the motion change. This allows us to assume that the viscoelastic and liquid components of the medium are continuous and at each point of space in this case there will be two displacement vectors, the displacement vector of the viscoelastic component (the skeleton of the porous medium) and the displacement vector of the liquid. We shall assume that the fluid is compressible.

Differential equations are obtained for determining the amplitude of longitudinal and transverse shock waves in a fluid-saturated inhomogeneous two-component viscoelastic medium using the mathematical theory of discontinuities [7] [8].

\section{Formulation Problem}

The interpenetrating motion of a viscoelastic component and a liquid is considered as a fluid motion in a deformable porous medium.

Rheological relations in such a medium through deformations will be written as

$$
T_{i j}=\Lambda^{*} e_{k k}^{(1)} \delta_{i j}+2 M^{*} e_{i j}^{(1)}+A_{1} e_{k k}^{(2)} \delta_{i j}, \quad N=A_{1} e_{k k}^{(1)}+A_{2} e_{k k}^{(2)}
$$

Here $\Lambda^{*}, M^{*}$ are linear integral operators whose relaxation kernels depend continuously on the spatial coordinates:

$$
\begin{aligned}
& \Lambda^{*}=\lambda(1+\tilde{\Lambda}), \tilde{\Lambda} e^{(1)}=\int_{0}^{\infty} \Lambda\left(t, x_{i}\right) e^{(1)}\left(t-t_{1}\right) \mathrm{d} t_{1} \\
& M^{*}=\mu(1+\tilde{M}), \tilde{M} e^{(1)}=\int_{0}^{\infty} M\left(t, x_{i}\right) e^{(1)}\left(t-t_{1}\right) \mathrm{d} t_{1}
\end{aligned}
$$

here $\lambda=\lambda\left(x_{i}\right), \quad \mu=\mu\left(x_{i}\right), \quad A_{1}\left(x_{i}\right)=(1-m) R_{0}, \quad A_{2}\left(x_{i}\right)=m R_{0}$ - continuous functions of coordinates, $m=m\left(x_{i}\right)$ porosity, $R_{0}=R_{0}\left(x_{i}\right)$-modulus of fluid compressibility, $T_{i j}$-of the stress tensor of a porous medium, $N$-force acting on the liquid per unit area of the cross section of the porous medium, $e_{i j}^{(1)}, e_{k k}^{(2)}$ - respectively, the components of the strain tensors of components, $\delta$-the Kronecker symbol. Index 1 above after the letter refers to the viscoelastic component, 2 to the liquid. 
Relations (1), (2) together with the equations of motion

$$
\begin{aligned}
& \rho_{11} \dot{V}_{i}^{(1)}+\rho_{12} \dot{V}_{i}^{(2)}=T_{i j, j}, \rho_{12} \dot{V}_{i}^{(1)}+\rho_{22} \dot{V}_{i}^{(2)}=N_{, i} \\
& \rho_{11}=\rho_{1}-\rho_{12}, \quad \rho_{22}=\rho_{2}-\rho_{12}
\end{aligned}
$$

and Cauchy formulas

$$
2 e_{i j}^{(1)}=u_{i, j}^{(1)}+u_{j, i}^{(1)}, \quad e_{k k}^{(2)}=u_{k, k}^{(2)}
$$

represent a closed system for describing the dynamic behavior of a fluid-saturated inhomogeneous two-component medium.

In formulas (3) and (4), $\rho_{11}=\rho_{11}\left(x_{i}\right)$-the effective density of the viscoelastic component, the $\rho_{22}=\rho_{22}\left(x_{i}\right)$-effective density of the fluid, $\rho_{12}=\rho_{12}\left(x_{i}\right)$ - the coefficient of dynamic coupling of the viscoelastic component and the liquid, $\rho_{1}\left(x_{i}\right), \rho_{2}\left(x_{i}\right)$ - and the density of the viscoelastic component and liquid, respectively.

The repeated Latin indices assume a summation of one to three, in Greek-from one to two. The dot above the letter indicates the time derivative, and the comma below the letter is the derivative of the coordinate.

\section{Velocities of Shock Waves}

A shock wave in an inhomogeneous two-component medium saturated with a liquid is determined by an isolated surface $\sum(t)$ on which the $u_{i}^{(\alpha)}(\alpha=1,2)$ displacements are continuous in time and coordinates, and the stresses $T_{i j}$ for ces $N$, and velocities of phase $V_{i}^{(\alpha)}(\alpha=1,2)$ discontinuities undergo. The parameters of the porous medium and their gradients are continuous.

Dynamic relationships must be satisfied on the wave surface $\sum(t)$ of a two-component medium [9]

$$
\left[T_{i j}\right] v_{j}=-\rho_{11} c\left[V_{i}^{(1)}\right]-\rho_{12} c\left[V_{i}^{(2)}\right],[N] v_{i}=-\rho_{12} c\left[V_{i}^{(1)}\right]-\rho_{22} c\left[V_{i}^{(2)}\right]
$$

where $c\left(x_{i}, t\right)$-the normal velocity of propagation of the surface, the $v_{i}$ - components of the unit normal to the surface, directed into the unperturbed region, which depend on the spatial coordinates.

The sign [ ] denotes the difference in the values of a certain quantity on different sides of the rupture surface.

From the rheological relations (1) and (2) written in the discontinuities, dynamic (5) and kinematic conditions of first-order compatibility for the phases, we obtain the system of equations

$$
\begin{aligned}
& \left\{\left(\rho_{11} c^{2}-\mu\right) \delta_{i j}-(\lambda+\mu) v_{i} v_{j}\right\}\left[V_{j}^{(1)}\right]+\left(\rho_{12} c^{2} \delta_{i j}-A_{1} v_{i} v_{j}\right)\left[V_{j}^{(2)}\right]=0 \\
& \left(\rho_{12} c^{2} \delta_{i j}-A_{1} v_{i} v_{j}\right)\left[V_{j}^{(1)}\right]+\left(\rho_{22} c^{2} \delta_{i j}-A_{2} v_{i} v_{j}\right)\left[V_{j}^{(2)}\right]=0
\end{aligned}
$$

Assuming that $\left[V_{j}^{(1)}\right]=S_{j}^{(1)} v_{j}=S_{1} \neq 0,\left[V_{j}^{(2)}\right]=S_{j}^{(2)} v_{j}=S_{2} \neq 0$ on the surface $\sum(t)$ where $S_{j}^{(\alpha)}(\alpha=1,2)$ the values of the discontinuities of the displacements of the components, multiply (6) by $v_{i}$ and sum over the repeating index $i$, we obtain a homogeneous system of equations for $S_{1}, S_{2}$. For sys- 
tem (6) to have a nonzero solution, its determinant must be zero. This condition leads to an equation for determining the propagation velocity of a longitudinal wave $\left(c=c_{p}\right)$

$$
\begin{gathered}
k c_{p}^{4}-k_{1} c_{p}^{2}+k_{2}=0, \quad k=\rho_{11} \rho_{22}-\rho_{12}^{2} \\
k_{1}=\rho_{11} A_{2}+\rho_{22} \Lambda_{1}-2 \rho_{12} A_{1}, \quad k_{2}=\Lambda_{1} A_{2}-A_{1}^{2}, \quad \Lambda_{1}=\lambda+2 \mu
\end{gathered}
$$

Equation (7), generally speaking, has two distinct roots, each of which determines the propagation velocity of the longitudinal wave $c_{p_{1}}$ and $c_{p_{2}}$

$$
c_{p_{1,2}}=\sqrt{\frac{k_{1} \pm \sqrt{k_{1}^{2}-4 k k_{2}}}{2 k}}
$$

Thus, in an inhomogeneous viscoelastic two-component medium, there are two types of longitudinal waves, which are determined by Formula (I).

On the other hand, if on the surface $\left[V_{j}^{(1)}\right] v_{j}=0,\left[V_{j}^{(2)}\right] v_{j}=0$, then from (6) it follows that the propagation velocity of the transverse wave $\left(c=c_{t}\right)$ is given by

$$
c_{t}=\sqrt{\frac{\mu \rho_{22}}{k}}
$$

Thus, it is shown that in a two-component inhomogeneous viscoelastic medium, there are three types of shock waves: two types of longitudinal waves and one transverse, for which, $\left[V_{i}^{(\alpha)}\right] v_{i}=S_{\alpha} \neq 0,\left[V_{i}^{(\alpha)}\right] v_{i}=0$ respectively, and the velocities of these waves are determined by Formulas (7) and (8).

If the connection between the viscoelastic component and the liquid is weak $\left(\rho_{12}=0, A_{1}=0\right)$, then from (7) and (8)

$$
c_{p_{1}}=\sqrt{\frac{\Lambda_{1}}{\rho_{11}}}=\sqrt{\frac{\lambda+2 \mu}{\rho_{1}}}, \quad c_{p_{2}}=\sqrt{\frac{A_{2}}{\rho_{2}}}, \quad c_{t}=\sqrt{\frac{\mu}{\rho_{11}}}=\sqrt{\frac{\mu}{\rho_{1}}}
$$

It follows from (9) that the propagation velocities of waves in a viscoelastic two-component medium are equal to the velocities of waves propagating separately in a continuous viscoelastic medium and liquid.

\section{Determination of Shock Wave Amplitudes}

Let us determine the change in the amplitude of the waves. To do this, we differentiate (1) with respect to $u$ and take their difference on different sides of the discontinuity surface, we obtain

$$
\begin{aligned}
{\left[\dot{T}_{i j}\right]=} & \lambda\left[V_{k, k}^{(1)}\right] \delta_{i j}+\mu\left(\left[V_{i, j}^{(1)}\right]+\left[V_{j, i}^{(1)}\right]\right)-\lambda \Lambda\left(0, x_{i}\right)\left[u_{k, k}^{(1)}\right] \delta_{i j} \\
& -\mu M\left(0, x_{i}\right)\left(\left[u_{i, j}^{(1)}\right]+\left[u_{j, i}^{(1)}\right]\right)+A_{1}\left[V_{k, k}^{(2)}\right] \delta_{i j}, \\
{[\dot{N}]=} & A_{1}\left[V_{k, k}^{(1)}\right]+A_{2}\left[V_{k, k}^{(2)}\right]
\end{aligned}
$$

Taking into account the compatibility conditions of the first order [6] and the equations of motion written in the discontinuities, (10) take the following form 


$$
\begin{aligned}
\frac{\delta\left[T_{i j}\right]}{\delta t}-M_{i j} c= & \lambda\left(L_{k}^{(1)} v_{k}+g^{\alpha \beta}\left[V_{k}^{(1)}\right]_{, \alpha} x_{\beta}^{k}\right) \delta_{i j}+\mu\left\{L_{i}^{(1)} v_{j}+L_{j}^{(1)} v_{i}\right\} \\
& +\mu g^{\alpha \beta}\left(\left[V_{i}^{(1)}\right]_{\alpha} x_{\beta}^{j}+\left[V_{j}^{(1)}\right]_{, \alpha} x_{\beta}^{i}\right)+\left\{\lambda \Lambda\left(0, x_{i}\right)\left[V_{k}^{(1)}\right] v_{k} \delta_{i j}\right. \\
& \left.+\mu M\left(0, x_{i}\right)\left(\left[V_{i}^{(1)}\right] v_{j}+\left[V_{j}^{(1)}\right] v_{i}\right)\right\} c^{-1} \\
& +A_{1}\left(L_{k}^{(2)} v_{k}+g^{\alpha \beta}\left[V_{k}^{(2)}\right]_{, \alpha} x_{\beta}^{k}\right) \delta_{i j} \\
\frac{\delta[N]}{\delta t}-\chi c= & A_{1}\left(L_{k}^{(1)} v_{k}+g^{\alpha \beta}\left[V_{k}^{(1)}\right]_{, \alpha} x_{\beta}^{k}\right)+A_{2}\left(L_{k}^{(2)} v_{k}+g^{\alpha \beta}\left[V_{k}^{(2)}\right]_{, \alpha} x_{\beta}^{k}\right) \\
-\rho_{11} c L_{i}^{(1)}+\rho_{11} & \frac{\delta\left[V_{i}^{(1)}\right]}{\delta t}-\rho_{12} c L_{i}^{(2)}+\rho_{12} \frac{\delta\left[V_{i}^{(2)}\right]}{\delta t}=M_{i j} v_{i}+g^{\alpha \beta}[N]_{, \alpha} x_{\beta}^{j} \\
-\rho_{12} c L_{i}^{(1)}+\rho_{12} & \frac{\delta\left[V_{i}^{(1)}\right]}{\delta t}-\rho_{22} c L_{i}^{(2)}+\rho_{22} \frac{\delta\left[V_{i}^{(2)}\right]}{\delta t}=\chi v_{i}+g^{\alpha \beta}[N]_{, \alpha} x_{\beta}^{i}
\end{aligned}
$$

The quantities, $M_{i j}, L_{i}^{(\alpha)}(\alpha=1,2), \chi$ and are determined on the surface $\sum(t)$ and characterize the jumps of the first derivative of the stresses, the forces acting on the fluid and the velocity of displacement of the components, respectively, $g^{\alpha \beta}$-the coefficients of the first fundamental quadratic form of the surface, $x_{\beta}^{i}$-the derivatives of the Cartesian coordinates $x_{i}$ along the curvilinear coordinates $y_{\beta}$ and $\delta / \delta t-\delta$-the time derivative.

By the standard method, we exclude the quantities $M_{i j}, \chi$ from (11). As a result, we get

$$
\begin{aligned}
& \rho_{11} c \frac{\delta\left[V_{i}^{(1)}\right]}{\delta t}+\rho_{12} c \frac{\delta\left[V_{i}^{(2)}\right]}{\delta t}-\rho_{11} c^{2} L_{i}^{(1)}+\lambda\left(L_{k}^{(1)} v_{k} v_{i}+g^{\alpha \beta}\left[V_{k}^{(1)}\right]_{, \alpha} x_{\beta}^{k} v_{i}\right) \\
& +\mu\left\{L_{i}^{(1)}+L_{k}^{(1)} v_{k} v_{i}+g^{\alpha \beta}\left[V_{k}^{(1)}\right]_{, \alpha} x_{\beta}^{i} v_{k}\right\}+\lambda \Lambda\left(0, x_{i}\right) c^{-1}\left[V_{k}^{(1)}\right] v_{k} v_{i} \\
& +\mu M\left(0, x_{i}\right) c^{-1}\left(\left[V_{i}^{(1)}\right]+\left[V_{k}^{(1)}\right] v_{k} v_{i}\right)+A_{1}\left(L_{k}^{(2)} v_{k} v_{i}+g^{\alpha \beta}\left[V_{k}^{(2)}\right]_{, \alpha} x_{\beta}^{k} v_{i}\right) \\
& -g^{\alpha \beta}\left[T_{i k}\right]_{, \alpha} c x_{\beta}^{k}-\frac{\delta\left[T_{i k}\right]}{\delta t} v_{k}=0 \\
& A_{1}\left(L_{k}^{(1)} v_{k} v_{i}+g^{\alpha \beta}\left[V_{k}^{(1)}\right]_{, \alpha} x_{\beta}^{k} v_{i}\right)+A_{2}\left(L_{k}^{(2)} v_{k} v_{i}+g^{\alpha \beta}\left[V_{k}^{(2)}\right]_{, \alpha} x_{\beta}^{k} v_{i}\right) \\
& -\rho_{12} c^{2} L_{i}^{(1)}+\rho_{12} c \frac{\delta\left[V_{i}^{(1)}\right]}{\delta t}-\rho_{22} c^{2} L_{i}^{(2)}+\rho_{22} c \frac{\delta\left[V_{i}^{(2)}\right]}{\delta t} \\
& -g^{\alpha \beta}[N]_{, \alpha} c x_{\beta}^{i}-\frac{\delta[N]}{\delta t} v_{i}=0
\end{aligned}
$$

From the rheological relations (1) written in the discontinuities, the dynamic (5) and kinematic conditions of first-order compatibility, we find, $\left[T_{i k}\right]_{, \alpha}$, $[N]_{, \alpha}, \frac{\delta\left[T_{i k}\right]}{\delta t} v_{k}, \frac{\delta[N]}{\delta t} v_{i}$, and substitute in (12)-(13). After the transformations, we obtain a system of equations determining the change in the components of the wave amplitude vector $\left(\left[V_{i}^{(\alpha)}\right]=S_{i}^{(\alpha)}, \alpha=1,2\right)$ 


$$
\begin{aligned}
& \left\{(\lambda+\mu) v_{k} v_{I}+\left(\mu-\rho_{11} c^{2}\right) \delta_{i k}\right\} L_{k}^{(1)}+\left(A_{1} v_{k} v_{i}-\rho_{12} c^{2} \delta_{i k}\right) L_{k}^{(2)}+2 \rho_{11} c \frac{\delta S_{i}^{(1)}}{\delta t} \\
& +2 \rho_{12} c \frac{\delta S_{i}^{(2)}}{\delta t}+\rho_{11} \frac{\delta c}{\delta t} S_{i}^{(1)}+\rho_{12} \frac{\delta c}{\delta t} S_{i}^{(2)}+\frac{1}{c}\left\{\left(\lambda \Lambda\left(0, x_{i}\right)+\mu M\left(0, x_{i}\right)\right) v_{k} v_{i}\right. \\
& \left.+\mu M\left(0, x_{i}\right) \delta_{i k}\right\} S_{k}^{(1)}+(\lambda+\mu) g^{\alpha \beta} S_{k, \alpha}^{(1)} x_{\beta}^{i} v_{k}+(\lambda+\mu) g^{\alpha \beta} S_{k, \alpha}^{(1)} x_{\beta}^{k} v_{i}-2 \mu \Omega S_{i}^{(1)} \\
& +\lambda_{, \alpha} g^{\alpha \beta} x_{\beta}^{i} S_{\beta}^{(1)} v_{k}+\lambda g^{\alpha \beta} x_{\beta}^{i} S_{k}^{(1)} v_{k, \alpha}+g^{\alpha \beta} x_{\beta}^{k}\left(\mu S_{k}^{(1)} v_{i, \alpha}+\mu_{, \alpha} S_{k}^{(1)} v_{i}\right) \\
& +A_{1} g^{\alpha \beta} S_{k, \alpha}^{(2)} x_{\beta}^{k} v_{i}+g^{\alpha \beta} x_{\beta}^{i}\left(A_{1, \alpha} S_{k}^{(2)}+A_{1} S_{k, \alpha}^{(2)} v_{k}+A_{1} S_{k}^{(2)} v_{k, \alpha}\right)=0 \\
& \left.+A_{1} v_{k} v_{i}-\rho_{12} c^{2} \delta_{i k}\right) L_{k}^{(1)}+2 \rho_{12} c \frac{\delta S_{i}^{(1)}}{\delta t}+\rho_{12} \frac{\delta c}{\delta t} S_{i}^{(1)}+A_{1} g^{\alpha \beta} S_{k, \alpha} x_{\beta}^{k} v_{i} \\
& +\left(A_{2} v_{k} v_{i}-\rho_{22} c^{2} \delta_{i k}\right) L_{k}^{(2)}+2 \rho_{22} c \frac{\delta S_{i}^{(2)}}{\delta t}+\rho_{22} \frac{\delta c}{\delta t} S_{i}^{(2)} \\
& \quad-g^{\alpha \beta} x_{\beta}^{i}\left\{\rho_{12} c c_{, \alpha} S_{k}^{(1)} v_{k}+\rho_{22} c c_{, \alpha} S_{k}^{(2)} v_{k}-\left(A_{1} S_{k}^{(1)} v_{k}\right)_{, \alpha}-\left(A_{2} S_{k}^{(2)} v_{k}\right)_{, \alpha}\right\}=0
\end{aligned}
$$

where $\Omega$-the average curvature of the wave surface.

From the relations (14) and (15) we obtain differential equations for determining the change in the amplitude of the longitudinal and transverse waves $\left(\left[V_{i}^{(\alpha)}\right] v_{i}=S_{i}^{(\alpha)} v_{i} \neq 0, S_{p}^{(\alpha)}=\sqrt{S_{i}^{(\alpha)} S_{i}^{(\alpha)}},\left[V_{i}^{(\alpha)}\right] v_{i}=0, \alpha=1,2\right)$.

For longitudinal waves, we multiply (14) and (15) by $v_{i}$ and sum over the repeated indices. As a result, we get

$$
\begin{aligned}
& \left(\Lambda_{1}-\rho_{11} c_{p}^{2}\right) L_{i}^{(1)} v_{i}+\left(A_{1}-\rho_{12} c_{p}^{2}\right) L_{i}^{(2)} v_{i}+\rho_{11} \frac{\delta c}{\delta t} S_{p}^{(1)}+2 \rho_{11} c_{p} \frac{\delta S_{p}^{(1)}}{\delta t} \\
& -2 \Lambda_{1} \Omega_{p} S_{p}^{(1)}+\frac{1}{c_{p}}\left(\lambda \Lambda\left(0, x_{i}\right)+2 \mu M\left(0, x_{i}\right)\right) S_{p}^{(1)}+\rho_{12} \frac{\delta c}{\delta t} S_{p}^{(2)} \\
& +2 \rho_{12} c_{p} \frac{\delta S_{p}^{(2)}}{\delta t}-2 A_{1} \Omega_{p} S_{p}^{(2)}=0, \Lambda_{1}=\lambda+2 \mu \\
& \left(A_{1}-\rho_{12} c_{p}^{2}\right) L_{i}^{(1)} v_{i}+\left(A_{2}-\rho_{22} c_{p}^{2}\right) L_{i}^{(2)} v_{i}+2 c_{p}\left(\rho_{12} \frac{\delta S_{p}^{(1)}}{\delta t}+\rho_{22} \frac{\delta S_{p}^{(2)}}{\delta t}\right) \\
& +\frac{\delta c_{p}}{\delta t}\left(\rho_{12} S_{p}^{(1)}+\rho_{22} S_{p}^{(2)}\right)-2 \Omega_{p}\left(A_{1} S_{p}^{(1)}+A_{2} S_{p}^{(2)}\right)=0
\end{aligned}
$$

We eliminate the quantity $L_{i}^{(2)}$ from (16) and (17). To do this, we multiply (16) by, $A_{2}-\rho_{22} c_{p}^{2}$ and (17) by $A_{1}-\rho_{12} c_{p}^{2}$ and subtract, we obtain the following equation of the first component of the two-component medium

$$
\begin{aligned}
& \frac{\delta S_{p}^{(1)}}{\delta t}=\left\{\Omega_{p} c_{p}-\frac{\beta_{2}}{2 c_{p}} \cdot \frac{\delta c_{p}}{\delta y}-\frac{A_{1}-\rho_{12} c_{p}^{2}}{2 c_{p}^{2} a_{1}}\left(\lambda \Lambda\left(0, x_{i}\right)+2 \mu M\left(0, x_{i}\right)\right)\right\} S_{p}^{(1)} \\
& a_{1}=\left(\rho_{11} \rho_{12} A_{2}+\rho_{12} \rho_{22} \Lambda_{1}-2 \rho_{11} \rho_{22} A_{1}\right) c_{p}^{2}-2 \rho_{12} A_{2} \Lambda_{1}+\rho_{22} A_{1} \Lambda_{1}+\rho_{11} A_{1} A_{2} \\
& \beta_{1}=\left(\rho_{11} A_{1}-\rho_{12} \Lambda_{1}\right)\left(\rho_{12} A_{2}-\rho_{22} A_{1}\right), \beta_{2}=\frac{A_{1} a_{1}+\left(4 \beta_{1}-\rho_{12} a_{1}\right) c_{p}^{2}}{a_{1}\left(A_{1}-\rho_{12} c_{p}^{2}\right)}
\end{aligned}
$$

When Equation (18) was obtained, the first Equation (6) and Formula (7) were used. 
For the transverse wave from (14) and (15), taking into account the second Equation (6) and Formula (8), we similarly obtain

$$
\frac{\delta S_{t}^{(1)}}{\delta t}=\left(\Omega_{t} c_{t}-\frac{1}{2 c_{t}} \cdot \frac{\delta c_{t}}{\delta t}-\frac{M\left(0, x_{i}\right)}{2}\right) S_{t}^{(1)}
$$

Passing to the variable $\sigma \geq 0$ denoting the distance along the normals to the surface $\sum\left(t_{0}\right)$ in (18) and (19), we obtain equations for changing the amplitude of the longitudinal and transverse waves during their propagation in the first component

$$
\frac{\mathrm{d} S_{l}^{(1)}}{\mathrm{d} \sigma}=\left\{\Omega_{l}-\chi_{1} \frac{\mathrm{d} \ln c_{l}}{2 \mathrm{~d} \sigma}-\chi_{2}\right\} S^{(1)}, \quad l=p, t
$$

where $\chi_{1}=\beta_{2}$-for longitudinal waves; $\chi_{1}=1$ for a transverse wave, $\chi_{2}=\frac{A_{1}-\rho_{12} c_{l}^{2}}{c_{l}^{2} a_{1}}(\lambda \Lambda(0, \sigma)+2 \mu M(0, \sigma))$-for longitudinal waves; $\chi_{2}=M(0, \sigma)$ - for a transverse wave.

The change in the amplitude of the waves in the second component is obtained from (6)

$$
S_{l}^{(2)}=\Gamma_{\alpha} S_{l}^{(1)}(\alpha=1,2 ; l=p, t), \quad \Gamma_{1}=\frac{\rho_{11} c_{p}^{2}-\Lambda_{1}}{A_{1}-\rho_{12} c_{p}^{2}}, \quad \Gamma_{2}=-\frac{\rho_{12}}{\rho_{22}}
$$

Then the change in the amplitude of the waves during their propagation in a porous medium will be written in the form

$$
S_{l}=S_{l}^{(1)}+S_{l}^{(2)}=\left(1+\Gamma_{\alpha}\right) S_{l}^{(1)}
$$

Equations (20) contain mean curvature $\Omega_{l}$, which is a function of $\sigma$.

The average curvature $\Omega_{l}(\sigma)$ is related to the first $g^{\alpha \beta}$ and second $b^{\alpha \beta}$ quadratic forms and the Gaussian curvature $K_{l}(\sigma)$ of the wave surface by the equations [10], where its method of finding is given.

The amplitude $S_{l}^{(1)}(l=p, t)$ level satisfying Equation (20) is found by the method of successive approximations under the initial conditions, $S^{(0)}(0)=S_{0}^{(0)}$, $S^{(i)}(0)=0 \quad(i=1,2, \cdots)$-approximation. A homogeneous medium corresponds to the zeroth approximation. In the first approximation, the rate of change in the inhomogeneity of the viscoelastic two-component medium along the beam is taken into account, and in the second, across the beam.

A more detailed solution of equations of the form (20) by the method of successive approximations was considered in [9] [10].

\section{Example}

We consider an inhomogeneous viscoelastic two-component medium saturated with a liquid, characterized by parameters and relaxation nuclei (a special case)

$$
\begin{aligned}
& \Lambda_{1}(x)=0.7 x^{2}, \rho_{11}(x)=0,6 x, \rho_{12}(x)=-0.1 x, \rho_{22}(x)=0.6 x, \\
& m(x)=0.5 x, R_{0}(x)=0.2 x, \Lambda(0, x)+2 M(0, x)=0.7 x
\end{aligned}
$$

At the moment of time $t=0$ in the plane $x y$ along the axis $O X$, the wave 
front propagates with velocity $c_{p}$ and $c_{t}$, which is determined from (7) (8) with $x_{i}=x$.

$$
\begin{gathered}
c_{p_{1}}=0.7 \sqrt{x}, c_{p_{2}}=0.45 \sqrt{x} \\
c_{t}=0.87 \sqrt{x}
\end{gathered}
$$

Since by the assumption of the problem the wave surfaces form a family of parallel planes, then the average curvature $\Omega_{l}=0$ at all times.

Then from (20) for, we obtain the dependence of the level of the wave amplitude on the velocity, the physico-mechanical characteristics and the relaxation kernels of the viscoelastic two-component medium for the first phase

$$
S_{p}^{(1)}=S_{0 p}^{(1)} \exp \left(-\int_{0}^{x}\left[\beta_{2}(x) \frac{\mathrm{d} \ln \sqrt{c(x)}}{\mathrm{d} x}+\frac{(1-0.5 x) 0.2+0.1 \cdot c^{2}(x)}{2 a_{1}(x) c^{3}(x)} \cdot 0.17 x^{3}\right] \mathrm{d} x\right)
$$

where $S_{0 p}^{(1)}$ the value of the function $S_{p}^{(1)}$ for $x=0, \beta_{2}(x)$, и $a_{1}(x)$ and are from (18).

By the formula (22) we obtain

$$
S_{p}=S_{0 p}\left(1+\Gamma_{1}(x)\right) S_{p}^{(1)}(x), \quad \Gamma_{1}(x)=\frac{\rho_{11}(x) c^{2}(x)-(\lambda(x)+2 \mu(x))}{(1-m(x)) R_{0}(x)-\rho_{12}(x) c^{2}(x)}
$$

Expression (25) determines the change in the level of the wave amplitude in a fluid-saturated inhomogeneous viscoelastic two-component medium.

If we specify in (25) a specific form of the physico-mechanical characteristics of the medium and the relaxation nuclei, we obtain an expression for determining the change in the level of the wave velositi in a fluid-saturated inhomogeneous viscoelastic two-component medium.

\section{Conclusions}

Applying the mathematical theory of discontinuities to the basic equations of a two-component viscoelastic porous medium, formulas were first obtained for determining the velocities and changing the level of the amplitude of longitudinal and transverse waves.

In the absence of dynamic coupling between the propagation velocity components of the first and second types of longitudinal waves, the wave velocities propagating separately in a continuous viscoelastic component and a continuous liquid component are equal.

In the medium under consideration, in the absence of a connection between the viscoelastic component and the liquid, the transverse wave propagates only in a continuous viscoelastic component.

Using geometric and dynamic conditions of second-order compatibility of the theory of discontinuities, equations are obtained for determining shock wave amplitudes in a saturated inhomogeneous viscoelastic two-component medium.

The amplitude of the waves in an inhomogeneous viscoelastic two-component medium depends on the porosity of the medium, the viscosity, the initial mean curvature, and the Gaussian curvature of the wave surface. 


\section{References}

[1] Rossikhin, Yu.A. and Meshkov, S.I. (1969) Propagation of Shock Waves in Linear Hereditary Media. Sat. Some Problems of Solid State Physics. News of the State Pedagogical University, 1, 49-53.

[2] Blitshtein, Yu.M., Meshkov, S.I. and Chigarev, A.V. (1972) Propagation of Waves in a Linear Viscoelastic Inhomogeneous Medium. Izvestiya AN SSSR. Mechanics of a Solid Body, № 3, 40-47.

[3] Polenov, V.S. (2014) Propagation of Elastic Waves in a Porous Medium Saturated with a Viscous Liquid. Applied Mathematics and Mechanics, 78, 501-507.

[4] Biot, M.A. (1956) Theory Propagation of Elastic Waves in a Fluid-Saturated Porous Solid I. Low-Frequency Range. The Journal of the Acoustical Society of America, 28, 168-178. https://doi.org/10.1121/1.1908239

[5] Biot, M.A. (1956) Theory Propagation of Elastic Waves in a Fluid-Saturated Porous Solid. II. Higher Frequency Range. The Journal of the Acoustical Society of America, 28. 179-191. https://doi.org/10.1121/1.1908241

[6] Kosachevsky, L.Ya. (1959) On the Propagation of Elastic Waves in Two-Component Media. Applied Mathematics and Mechanics, 23, 1115-1123.

[7] Bykovtsev, G.I. and Verveiko, N.D. (1966) On the Propagation of Waves by an Elastic-Viscous-Plastic Medium. Mechanics of a Solid, № 4, 111-123.

[8] Thomas, T.Y. (1961) Plastic Flow and the Fracture in Solids. Academic Press, New York, $267 \mathrm{p}$.

[9] Polenov, V.S. and Chigarev, A.V. (2010) Propagation of Waves in an Inhomogeneous Porous Medium Saturated with a Liquid. Applied Mathematics and Mechanics. 74, 276-284.

[10] Chigarev, A.V. (1980) On the Geometry of Wave Fronts in Inhomogeneous Media, Acoustic Journal, 26, 905-912.

\section{A List of Symbols}

$T_{i j}:$ stress tensor of a porous medium

$N$ : force acting on the liquid per unit area of the cross section of the porous medium

$e_{i j}^{(1)}$ : components of the strain tensors of the elastic component

$e_{k k}^{(2)}$ : components of fluid deformations

$u_{i}^{(1)}$ : displacement of the elastic component

$u_{1}^{(2)}$ : fluid movement

$\Lambda^{*}, M^{*}$ : linear elastic integral operators

$S_{P}^{(\alpha)}$ : wave amplitudes

$\Omega$ : average curvature of the wave surface

$g^{\alpha \beta}$ : the coefficients of the first fundamental quadratic form of the surface 